\title{
Features of Transient Flow during Collapse of Nuclear Power Pump Cavitation
}

\author{
Rongsheng Zhu, Xiuli Wang*, Qiang Fu and Yun Long \\ Research Center of Fluid Machinery Engineering and Technology, Jiangsu University, Zhenjiang, Jiangsu 212013, China
}

Received: 2 Jun. 2013, Revised: 7 Oct. 2013, Accepted: 8 Oct. 2013

Published online: 1 May. 2014

\begin{abstract}
Collapse of cavitation in flow passage of impeller for centrifugal pump was stimulated by CFX to numerically stimulate internal features of cavitation when pressure at entrance continuing to decline. Results of numerical simulation and tests presented consistent tendency. Cvol in the flow passage of impeller was zero for a while in the occurrence of cavitation collapse and then increased sharply. After reaching a certain value, Cvol began to increase slowly. The amplitudes of head fluctuations increased with decreasing flow within the interval prior to a specific value and then increased with increasing flow.
\end{abstract}

Keywords: Centrifugal pump, Transient flow, Cavitation, Numerical simulation, Collapse condition

\section{Introduction}

Cavitation is a complex non-steady hydrodynamic phenomenon. The generation and development of cavitation is an important research field on centrifugal pumps, which are main components of nuclear power pump. Cavitation of centrifugal pump is the process that the empty bubbles collapse in the impeller. This process will produce huge impulse on impeller and pump body, resulting in increasing vibration and large amplitude and even causing accident. Major researches on cavitation include: Alicja Krella etal [1,2,3,4] have carried out a large number of experiments on damage $4 \mathrm{~s}$ caused by cavitation and corrosion; Mirko et. al. [5,6,7,8,9] reported the changes of pressure pulsation and vibration in the process of cavitation. Researches on internal laws of cavitation mainly include: William A. [10] have studied the generation of cavitation; Xavier [11] have carried out researches on spiral cavitation. Many researchers have done a lot of work in the field of cavitation. However, these studies focused on features of steady cavitation and vibration induced by cavitation. No researches referring to features of transient flow during cavitation of centrifugal pump were found, especially researches on internal flow when $\mathrm{O}-\mathrm{H}$ steep drop in the occurrence of cavitation collapse.

Therefore, through review the results of previous studies, the transient computational model for collapse of cavitation were established. Non-steady features from critical NPSH to steep drop of QH during cavitation collapse of impeller of centrifugal pump was modeled by CFD numerical simulation. The calculation results were compared with experimental data to verify the reliability of the results in order to provide reference for studies on internal non-steady flow of centrifugal pump in the presence of cavitation.

\section{Numerical Simulations}

\subsection{Cavitation model}

The cavitation model is kind of mathematical model to describe the conversion between water and water vapor. The full cavitation model is used in this study and the effects of un-dissolved gas on cavitation were taken into account. The density of fluid was regarded as function of gas mass fraction in this model. Gas mass fraction can be used to solve transport equations, including both of mass equation and momentum equation. Generally, the area with low pressure is more subject to cavitation, where speed is relatively high. In such area, velocity slip between liquid and gas is quite small, which can be assumed to be zero. The converged solutions for liquid and cavitation bubble can be calculated from the mutual iteration of the two-phase turbulence considering inter-phase forces.

\footnotetext{
*Corresponding author e-mail: jsuwxl@163.com
} 


\subsection{Computational model and generation of grids}

Sample: volute centrifugal pump; performance parameters: the transmission medium is water, flow $\mathrm{Q}=35 \mathrm{~m} 3 / \mathrm{h}$, head $\mathrm{H}=32 \mathrm{~m}$, rotated speed $=2900 \mathrm{r} / \mathrm{min}$, specific speed $=70$, number of blades: 6. Threedimensional computational domain was generated by $\mathrm{PRO} / \mathrm{E}$. In order to achieve more stable results, the inlet of impeller was appropriately extended. The entire model consisted of a moving impeller water body, a stationary volute water body and the extended segment in the inlet. CFX pre-treatment software-ICEM was used to generate tetrahedral unstructured grid. The impeller was divided into 581014 grids. Volute was divided into 371367 grids. Then "smooth" function of ICEM was applied to smooth grids. The Three-dimensional computational domain was shown in Figure 1.
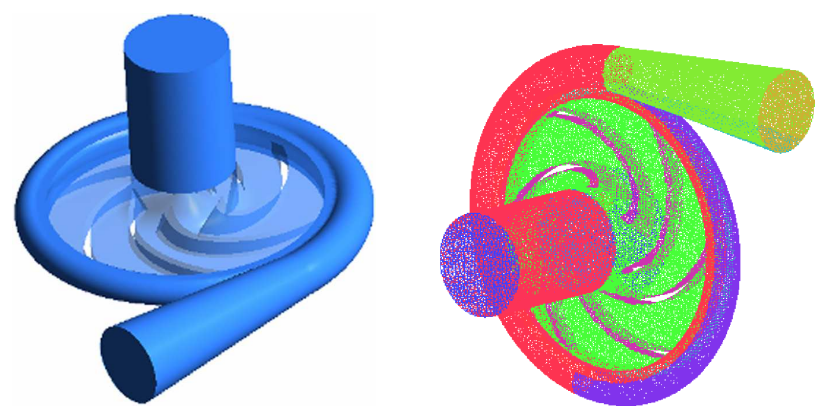

Fig. 1: Computational domain.

\subsection{Boundary conditions}

Inlet pressure conditions were set for the inlet of centrifugal pump. In order to ensure reliable results, internal flow was detected after running for $0.001724 \mathrm{~s}$. The "ce" of cfx was used to set the changes of pressure in inlet. The describing function was as follows:

$$
P(t)= \begin{cases}p_{a} & t<0.001724 \mathrm{~s} \\ p_{a}+p_{0}\left(t-t_{0}\right) & t \geq 0.001724 \mathrm{~s}\end{cases}
$$

Where: $\mathrm{P}(\mathrm{t})$ - inlet pressure, $\mathrm{Pa} ; \mathrm{P}_{a}$ - critical NPSH pressure, $\mathrm{Pa} ; \mathrm{t}$ - time, $\mathrm{s} ; \mathrm{t}_{0}$ — initial time, $0.001724 \mathrm{~s}$;

The mass flow in outlet was given in outlet conditions. Mass flow was controlled by control model of outlet boundary conditions. The roughness of wall was set to $10 \mu \mathrm{m}$; standard wall function is applicable for the region near wall; the boundary condition of wall was set to adiabatic non-slip wall; the average diameter of bubble was set to $2 \mathrm{e}-06 \mathrm{~m}$; the water volume fraction at inlet was set to 0 .

\subsection{Settings of non-steady simulation and selection of monitoring locations}

Water in impeller passage was rotating body, while water in volute was non-rotating body. Steady converged solution was regarded as initial condition for non-steady calculation. The interface of non-steady calculation was set to mode of Transient Rotor-Stator, which played an important role to dynamic-static interference between water bodies. The total computing time was 1s. Every turn of $3^{\circ}$ of impeller was as one time step. Time step was $1.724 \mathrm{e}-4 \mathrm{~s}$.

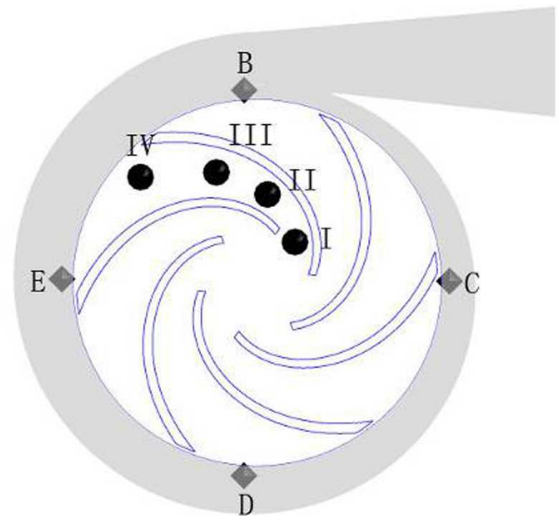

Fig. 2: Distribution of monitoring locations in pump.

Sites as shown in Figure 2 were selected to monitor internal pressure, velocity and gas pressure fraction. Four monitoring sites were arranged in the passage of impeller, naming I, II, III and IV; Four monitoring sites, i.e. B, C and $\mathrm{E}$ were located near the interface between volte and impeller. Data was collected every $90^{\circ}$ when each point was at the middle section. Such arrangement can fully monitor internal flow state in the occurrence of cavitation for centrifugal pump.

\section{Simulated Results and Analysis}

Transient modeling of cavitation collapse for centrifugal pump involves a series of continuous states over time. For the purpose of analyzing the changes of internal flow in the occurrence of cavitation, internal gas volume fraction of impeller and velocity vector at four time points $(0.035 \mathrm{~s}$, $0.05 \mathrm{~s}, 0.065 \mathrm{~s}$ and $0.8 \mathrm{~s}$ ) under rated working mode were analyzed.

\subsection{Changes of gas volume fraction}

Changes of gas volume fraction overt times were given in Figure 3. It can be seen from Figure 3 that the maximum gas volume fractions were mainly distributed in a particular region near the entrance of the back of impeller. 
Gas volume fractions were low near the entrance and then gradually increasing along outlet of impeller passage. In the view from radial direction, the gas volume fraction gradually increased from the back of blade. The changes of gas volume fraction varied among each passage. Gas volume fraction region accounted for one-third of the entire flow passage. When the inlet pressure was gradually decreasing, the gas volume fraction increased along out and began to block the entrance of impeller passage. Subject to separation by boundary layer, spiral vortex and cut water, gas volume fraction developed from arc-like to vertical line with blade and then became a straight line with a certain angle with the blade during the moving process in flow passage. The gas volume fraction almost occupied the entire flow passage, which seriously blocked internal energy exchange and transmission.

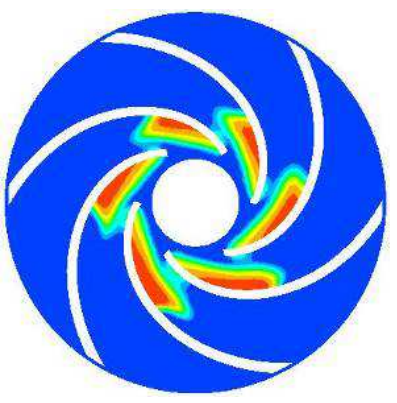

(a) $0.035 \mathrm{~s}$

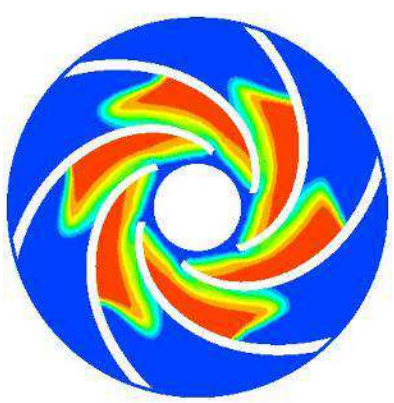

(c) $0.065 \mathrm{~s}$

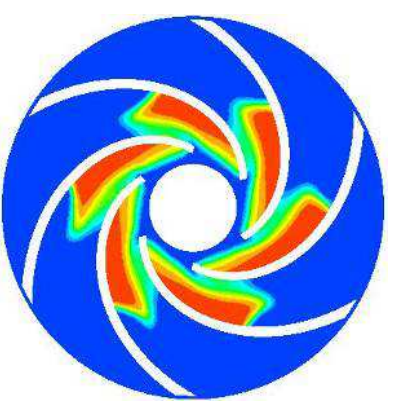

(b) $0.05 \mathrm{~s}$

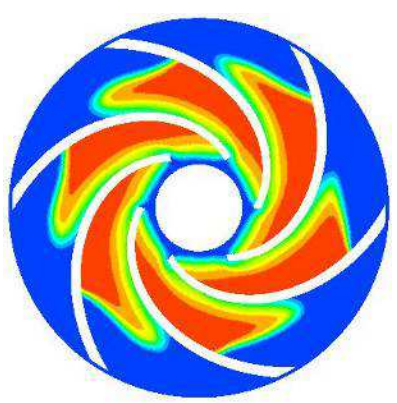

(d) $0.08 \mathrm{~s}$
Fig. 3: Gas volume fractions in passage of impeller.

\subsection{Changes of relative velocity}

The streamline cloud picture as shown in Figure 4 indicated the changes of internal relative velocity in impeller. It can be seen from the figure that small amount of spiral vortexes appeared close to middle positions of most of working face at $0.035 \mathrm{~s}$, indicating that the gas volume fraction has begun to affect the internal flow. Gas volume fraction still existed in the rest of flow passages but streamline was uniform and stable, which had little effect on internal flow; Boundary separation appeared near the middle position of back of blade. With decreasing pressure at entrance, the vortex near the blade face did not continue to grow but slowly moved to the out let of impeller. Vortex at the back of blade began to grow gradually; vortex at working face has gone at $0.08 \mathrm{~s}$ but large vortex occurred at back of blade near the outlet.

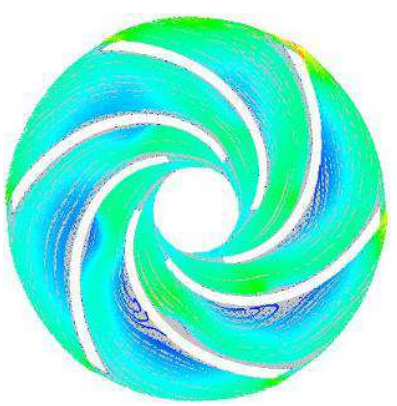

(a) $0.035 \mathrm{~s}$

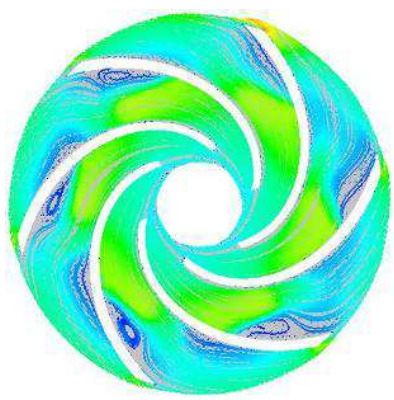

(c) $0.065 \mathrm{~s}$

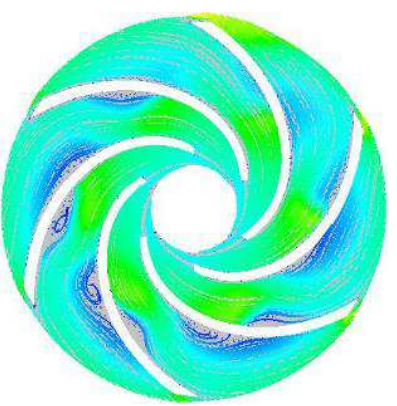

(b) $0.05 \mathrm{~s}$

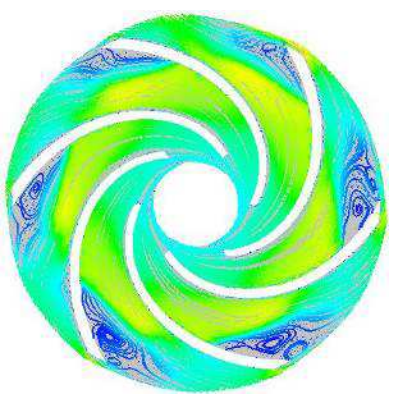

(d) $0.08 \mathrm{~s}$
Fig. 4: Changes of internal relative velocity in impeller.

\subsection{Changes of gas volume fraction for various monitoring locations in impeller}

Changes of gas volume fraction at various points in flow passage of impeller were shown in Figure 5. Cvol was defined as gas volume fraction. It can be seen from the figure that the initial Cvol for each point was zero, indicating none of points had cavitation at the beginning. Gas volume fraction Cvol at I sharply increased to a certain value within very short time at $0.18 \mathrm{~s}$ and then increased slowly; Values of Cvol at other points also began to sharply increase following with different Cvol gradients and maximum values.

\subsection{Changes of velocity various monitoring locations in impeller}

Figure 6 showed the changes of velocity in impeller. It can be seen that in general the velocity in impeller progressively increased. Velocities at point I and point II increased slightly before $0.3 \mathrm{~s}$ and $0.43 \mathrm{~s}$ and then remained constantly constants subsequently after achieving maximum, indicating that bubbles generated by cavitation did not completely filled until $0.3 \mathrm{~s}$ and $0.43 \mathrm{~s}$; velocity changed in volatility and flow rate was relatively 


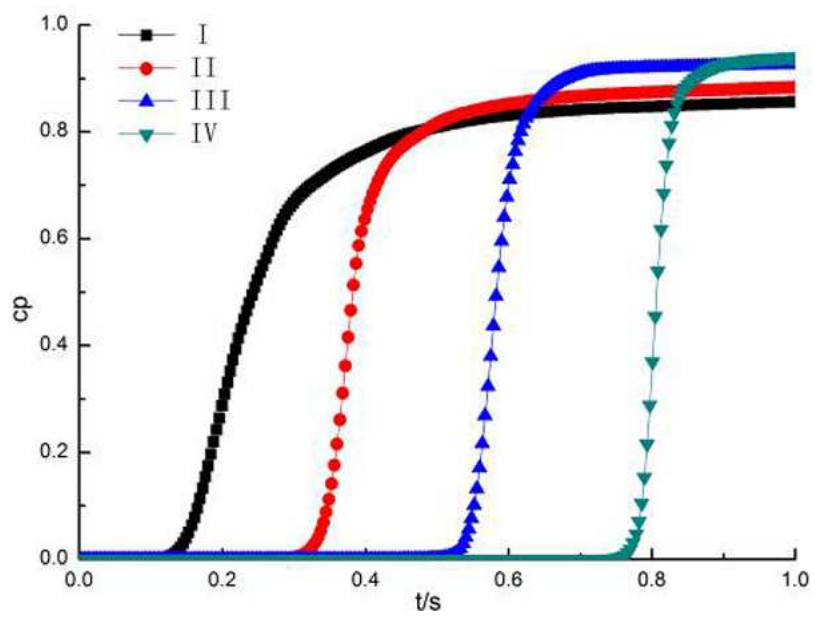

Fig. 5: Gas volume fractions in passage of impeller.

high due to crowding bubbles and vortex induced by cavitation so that flow rate in impeller passage increased and presented unstable situation. There were no big changes of velocity for point III and point IV at the beginning but the velocity fluctuated $0.5 \mathrm{~s}$ lately and then increased in a sudden. The reasons were indicated in Figure 4 that vortex occurred in the middle of flow passage. Vortex caused large fluctuations of velocity and also delayed the changes of velocity in impeller thus vortex moved towards the outlet of impeller, and made the velocity increased suddenly. Overall, vortex induced by cavitation had great impacts on velocity in impeller.

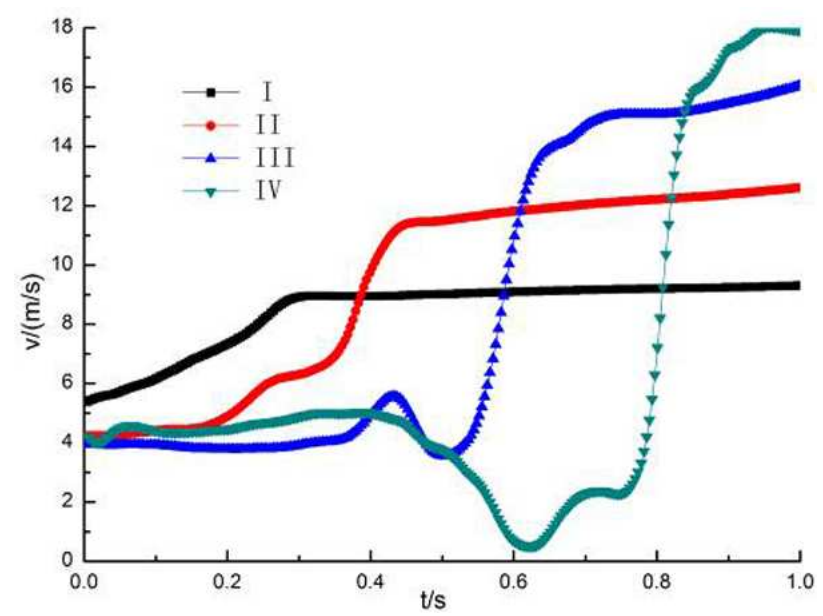

Fig. 6: Changes of relative velocity in impeller.

\subsection{Changes of pressure at various monitoring sites in impeller}

Pressure curve for different monitoring sites during collapse of cavitation was given in Figure 7. Comparing with large fluctuations of velocity in impeller, the variations of internal pressure are quite uniform. It can be seen from the figure that with growth of radial locations, the amplitude of pressure fluctuations was relatively large. Pressure at point I dropped from 4000pa to 150pa at within $0.3 \mathrm{~s}$ almost without any pressure fluctuation during this period. Pressures at other points such as point II fluctuated within small ranges.

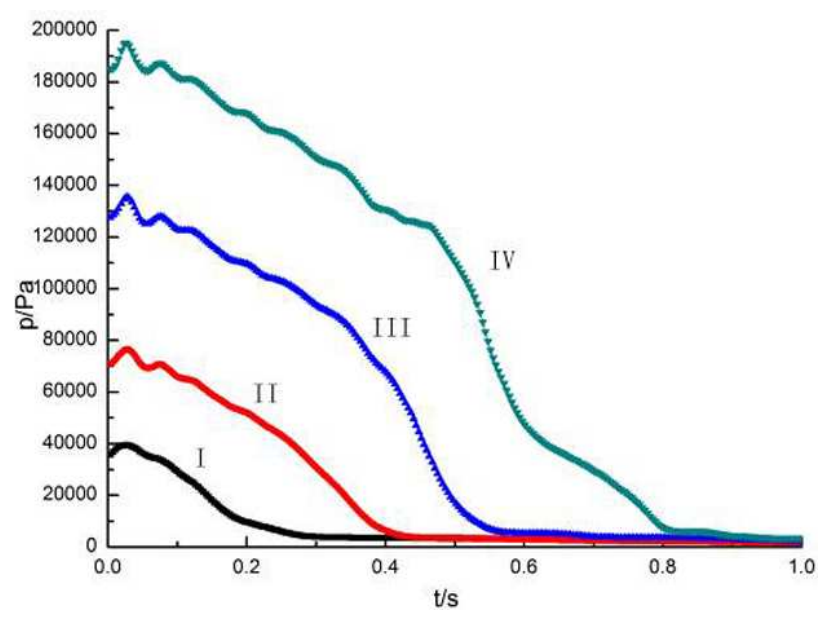

Fig. 7: Changes of relative pressure in impeller.

\subsection{Changes of speed at various monitoring sites in impeller}

Velocity curve for different monitoring sites during collapse of cavitation was given in Figure 8 . It can be seen from the figure that there were few changes of velocity for all monitoring sites before $0.6 \mathrm{~s}$ but velocity declined sharply $0.6 \mathrm{~s}$ later after a wide range of changes, indicating that cavitation had limited impacts on velocity at outlet of impeller during a while at critical NPSH. Cavitation played great impacts on velocity at outlet of impeller only in the occurrence of cavitation collapse.

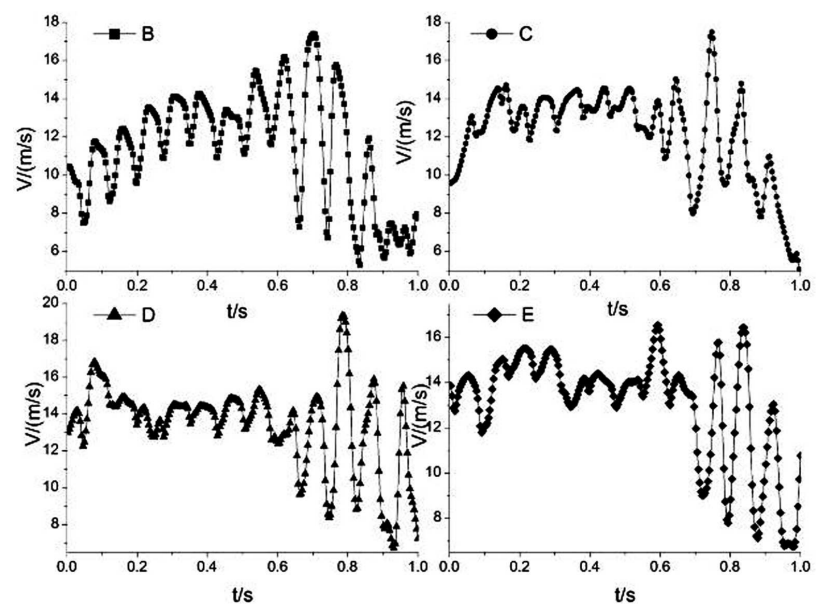

Fig. 8: Changes of relative velocity at outlet of impeller. 


\subsection{Changes of pressure at various monitoring sites in outlet of impeller}

Pressure curve for different monitoring sites during collapse of cavitation was given in Figure 9. It can be seen from the figure that the relative pressures at various monitoring sites in outlet of impeller presented same trend overall. Due to dynamic and static interference and the presence of vortex induced by cavitation, pressure changed in regularity at the beginning; however, pressure fluctuated irregularly since $0.5 \mathrm{~s}$, which was mainly due to the collapse of cavitation. Cavitation was serious at that time. Strong interaction between vapor and liquid resulted in pressure fluctuations.

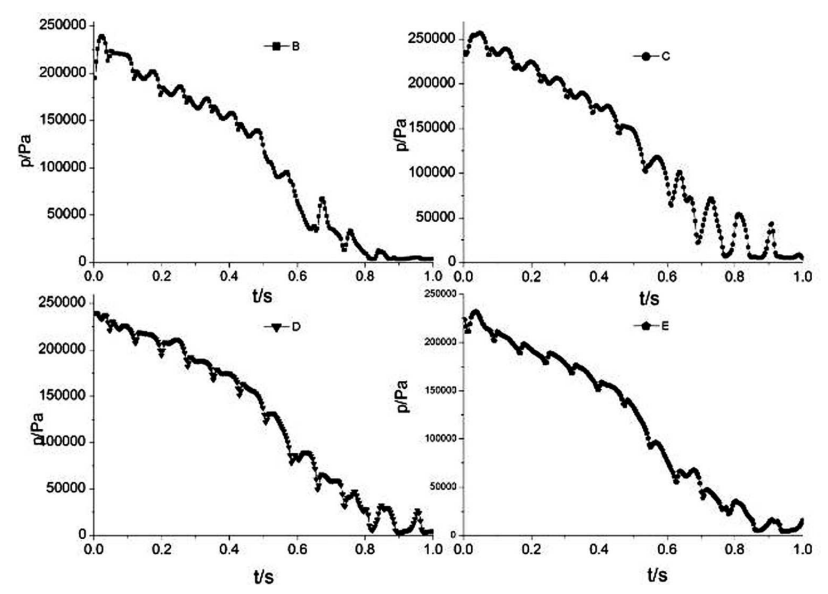

Fig. 9: Changes of relative pressure at outlet of impeller.

\subsection{Changes of head under different conditions}

Figure 10 showed the changes of head under different conditions in the occurrence of cavitation. It can be seen from the figure that head gradually declined over time or decreasing pressure at entrance. Changes of head under different conditions presented a same overall trend. The main differences were: pressure fluctuations of head increased with the reduction of flow before $0.6 \mathrm{~s}$ and then increased with increase of flow.

\section{Experimental Results and Analysis}

According to GB/T 3216-2005 Rotodynamic pumpsHydraulic Performance Acceptance Tests-Grade 1 and 2, cavitation test was carried out on test table of Grade B accuracy for centrifugal pump sample. The flow maintained constant and resistance was regulated by the valve of entrance. Through increasing resistance, the pressure at entrance of pump was reduced gradually. NPSH value was adjusted until head decreases amounted to $3 \%$ and NPSH at that time was determined as critical

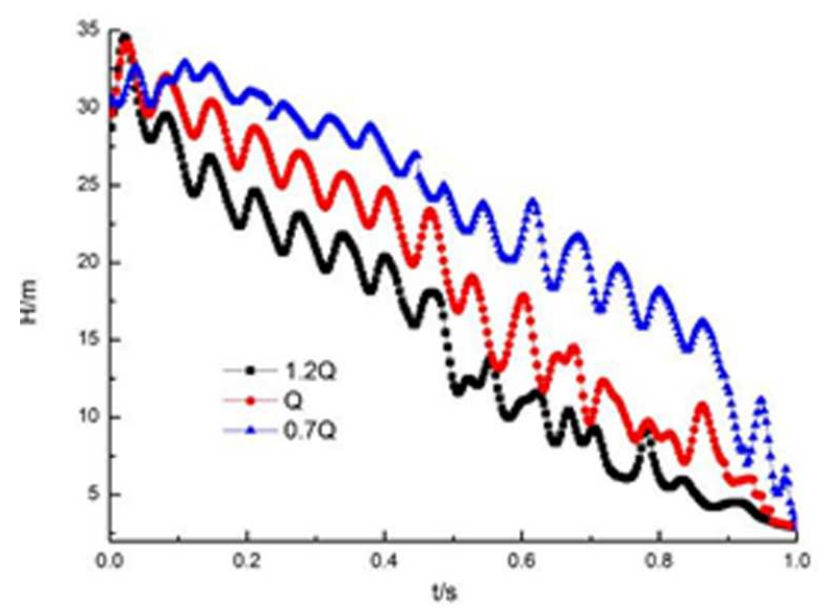

Fig. 10: Changes of head under different conditions.

cavitation margin. Then valve was further regulated until the occurrence of cavitation. The experimental data was shown in Figure 9.

Performance of cavitation under $0.7 \mathrm{Q}, \mathrm{Q}$ and $1.2 \mathrm{Q}$ (Q-rated condition) was given in Figure 9. It can be seen that the stimulated heads for corresponding NPSH were $34.8 \mathrm{~m}, 33.6 \mathrm{~m}$ and $30.35 \mathrm{~m}$ respectively and the test data were $33.6 \mathrm{~m}, 32.5 \mathrm{~m}$ and $29.5 \mathrm{~m}$ relatively with an absolute error of about $3 \%$. Therefore, there was no big difference between test results and stimulated results and both of then shared the similar overall tendency. The difference was resulted from casting, mechanical processing, surface roughness and boundary condition settings during simulation, grids number and cavitation model, etc. It can be believed that the numerical model and computational method were credible.

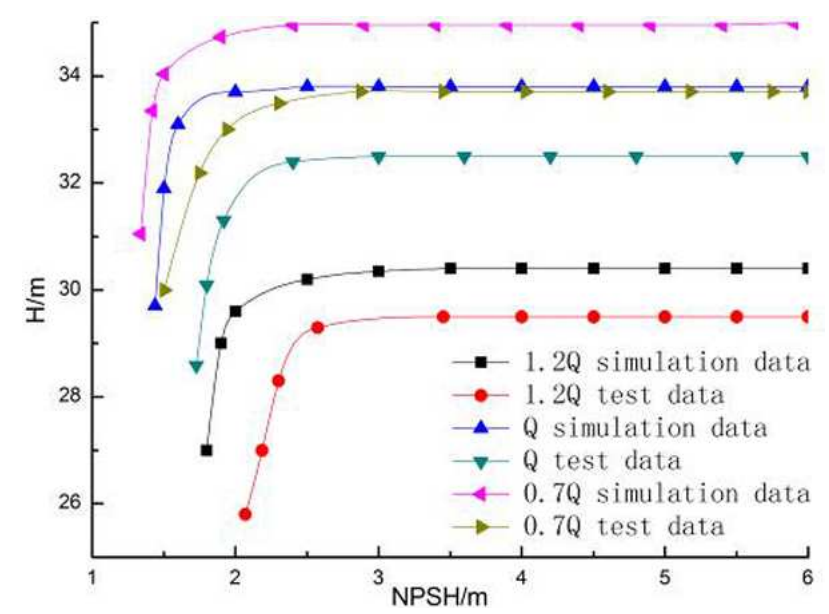

Fig. 11: Cavitation performance under different conditions.

\section{Conclusions}

Gas volume fraction Cvol in the flow passage of impeller was zero for a while at the beginning in the occurrence of 
cavitation collapse and then increased sharply. After reaching a certain value, Cvol began to increase slowly. The maximum of gas volume fraction for different sites were not the same.

There were no big changes of velocity during the beginning. After irregular and large changes, velocity dropped sharply.

At the beginning, vortex occurred near the middle of working face of blade. Boundary separation and vortex were shown near the middle of back of blade. With decreasing pressure at entrance, the vortex near working face moved towards outlet of impeller and disappeared, while the vortex at back of blade gradually developed.

Head decreased with declining pressure at entrance. The amplitudes of head fluctuations varied under different conditions. The amplitudes of head fluctuations increased with decreasing flow within the interval prior to a specific value and then increased with increasing flow.

\section{Acknowledgements}

This work is partially supported by National Science Found for Distinguished Young Scholars of China (50825902), National Science Found of Jiangsu Province (BK2009218), A Project Founded by the Priority Academic Program Development of Jiangsu Higher Education Institutions. Thanks for the help.

\section{References}

[1] Alicja Krella. An experimental parameter of cavitation erosion resistance for TiN coatings. Wear, 270, 3-4, 252257 (2011).

[2] Fei Ren, John Jy-An Wang, Hong Wang. Alternative approach for cavitation damage study utilizing repetitive laser pulses. Wear, 270, 115-119 (2010).

[3] S. M. Ahmed, K. Hokkirigawa, R. Oba. Fatigue failure of SUS 304 caused by vibratory cavitation erosion. Wear, 177, 129-137 (1994).

[4] S. F. Brunatto, A. N. Allenstein, C. L. M. Allenstein, A. J. A. Buschinelli Cavitation erosion behavior of niobium. Wear, 274-275, 220-228 (2012).

[5] Mirko Čudina, Jurij Prezelj. Detection of cavitation in situ operation of kinetic pumps: Effect of cavitation on the characteristic discrete frequency component. Applied Acoustics, 70, 1175-1182 (2009).

[6] Jin-ming YE, Ying XIONG, Fang LI, Shuang-qiao CHEN. Experimental study of effects of air content on cavitation and pressure fluctuations. Journal of Hydrodynamics, Ser. B, 22, 634-638 (2010).

[7] Yongyong He, Yuan Liu. Experimental research into timefrequency characteristics of cavitation noise using wavelet scalogram. Applied Acoustics, 72, 721-731 (2011).

[8] Xiang Wang, Wanhua Su. Numerical investigation on relationship between injection pressure fluctuations and unsteady cavitation processes inside high-pressure diesel nozzle holes. Fuel, 89, 2252-2259 (2010).
[9] Regiane Fortes Patella, Jean-Luc Reboud, Antoine Archer. Cavitation damage measurement by $3 D$ laser profilometry. Wear, 246, 59-67 (2000).

[10] William A. Straka, Richard S. Meyer, Arnold A. Fontaine, Joseph P. Welz. Cavitation inception in quiescent and coflow nozzle jets. Journal of Hydrodynamics, Ser. B, 22, 813819 (2010).

[11] Xavier Escaler, Philippe Dupont, François Avellan. Experimental investigation on forces due to vortex cavitation collapse for different materials. Wear, 233-235, 65-74 (1999).

[12] Kai-Long Hsiao. Multimedia Feature for Unsteady Fluid Flow over a Non-Uniform Heat Source Stretching Sheet with Magnetic Radiation Physical Effects. Appl. Math. Inf. Sci., 6 No. 1S, 59S-65S (2012).

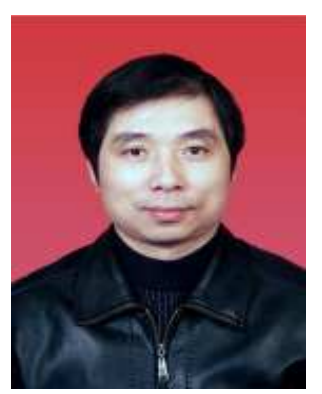

Rongsheng Zhu received the MS degree in Hydraulic Machinery from Huazhong University of Science and Technology in 1985, and the $\mathrm{PhD}$ degree in Hydraulic Machinery from Research Center of Fluid Machinery Engineering and Technology, Jiangsu University in 2011. $\mathrm{He}$ is currently a professor in Jiangsu University. His research interests are in the areas of pump design and manufacture, hydraulic machinery, and fluid engineering systems. 